\title{
EFFECT OF THE PULSATIONS GENERATED BY THE MAIN CIRCULATION PUMPS ON THE MEASUREMENT OF THE COOLANT FLOW IN THE VVER 1000/320 REACTOR
}

\author{
L. Pečínka* ,Vl. Krupa**
}

\begin{abstract}
Measurement of the coolant flow in the primary circuit of the VVER 1000/320 reactor is based on the measurement of the pressure drop either on the main circulation pump, or reactor or steam generator. It will be proved that the existence of the pressure pulsations result in differences between the rate indicated by the flow meters and the actual flow rate.
\end{abstract}

Keywords: pressure pulsations, sine wave, binomial theorem, trinomial expansion, frequency changes.

\section{Introduction}

The existence of the pressure pulsations in the coolant flow and its effect on flowmeters is well known. Lindahl (1946) published in the Transaction of the ASME overview of up the present time published papers. Another excellent survey of the publications was presented by Oppenheim (1955). For the incompressible flow using continuity equation and equation of motion the steady flow equation is derived and the problem is concentrated on the measurement of the pressure drop which is influenced by the pressure pulsations. In order to show how this pulsations act on the mathematical analysis will be in the next presented. It must be kept in the mind that the method of calculation of the error due to pulsations is only theoretical approach.

\section{Mathematical analysis of the pulsations effect}

The main circulation pumps (MCP) generate sine waves as shown in Fig. 1a, where $P_{0}$ represents nominal value of the pressure and $\Delta p$ amplitude of the pressure pulsations.

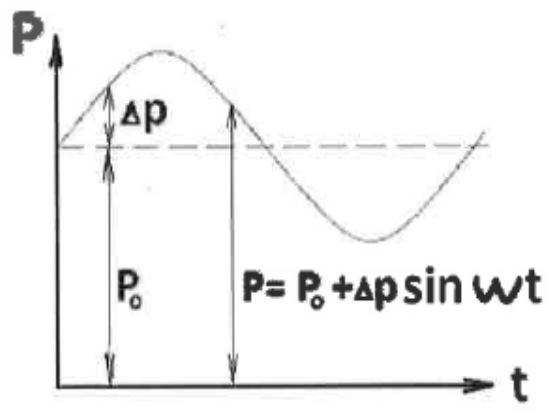

a

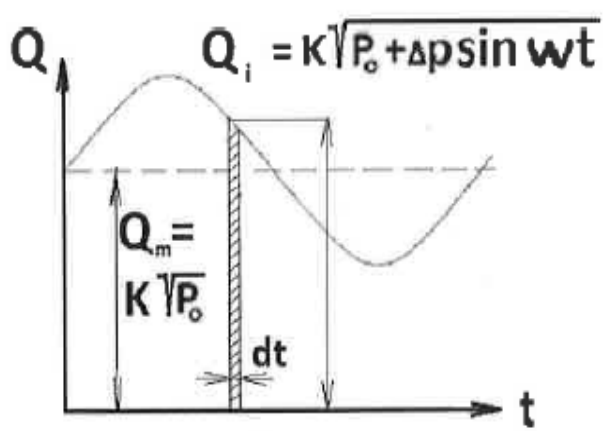

b

Fig. 1: a) Pressure generated by the main circulation pumps, b) flow rate in the moment $d t$

\footnotetext{
* Ing. Ladislav Pečínka, CSc., ÚJV Řež, a.s., Hlavní 130, Řež, 25068 Husinec; CZ; Ladislav.Pecinka@ujv.cz

** Prom. Fyz. Vladimír Krupa, ÚJV Řež, a.s., Hlavní 130, Řež, 25068 Husinec; CZ; Vladimir.Krupa@ujv.cz
} 
Instantaneous true rate of the flow is

$$
Q=\bar{K} \sqrt{\left(P_{0}+\Delta p \sin \omega t\right)}
$$

where $\omega$ is the frequency of the rotor revolutions and $\bar{K}=K A \sqrt{2 \rho}$. The flow rate in the time moment $\mathrm{d} t$ (see Fig.1b) is

$$
Q=\bar{K} \sqrt{\left(P_{0}+\Delta p \sin \omega t\right)} \mathrm{d} t
$$

and the total flow per one period of the pressure pulsations is

$$
Q=\bar{K} \int_{0}^{T} \sqrt{\left(P_{0}+\Delta p \sin \omega t\right)} \mathrm{d} t
$$

The average flow per one period is

$$
Q_{a}=\frac{\bar{K}}{T} \int_{0}^{T} \sqrt{\left(P_{0}+\Delta p \sin \omega t\right)} \mathrm{d} t
$$

To obtain numerical value of $Q_{a}$, the term $\sqrt{\left(P_{0}+\Delta p \sin \omega t\right)}$ must be expanded by the binomial theorem and after that integration performed.

\section{Application to the primary circuit of the VVER 1000/320 reactor}

In many cases the flow rate is obtained from the pressure drop on main circulation pumps, reactor and steam generator. The specific problems of this solution are described in the next.

\subsection{Measurement on the MCP}

The pressure drop is measured as a difference between pressure on the pump discharge and on the pump suction. It is well known that the MCP generate pressure pulsations. It means that the pressure on the pump discharge takes the form $P=P_{0}+\overline{\Delta p} \sin \left(\omega+\delta_{i}\right) t$ where $\overline{\Delta p}$ is the amplitude of the pressure pulsations, $\omega$ are the nominal angular frequency of the MCP rotor and $\delta_{i}$ represent deviation from the nominal angular frequency $\omega$ since the electrical motor is asynchronous. In this case equation (4) takes the form

$$
Q_{a}=\frac{\bar{K}}{T} \int_{0}^{T} \sqrt{\left(P_{01}+\overline{\Delta p} \sin \left(\omega+\delta_{i}\right) t-P_{02}\right)} \mathrm{d} t=\frac{\bar{K}}{T} \int_{0}^{T} \sqrt{\left(P_{01}-P_{02}\right)+\overline{\Delta p} \sin \left(\omega+\delta_{i}\right) t} \mathrm{~d} t
$$

where $P_{01}$ and $P_{02}$ are nominal values of the coolant flow in the MCP discharge and suction. Using binomial theorem and performing integration as the result we obtain

$$
\frac{Q_{M}}{Q_{a}}=\frac{K\left(P_{01}-P_{02}\right)^{\frac{1}{2}}}{\bar{K}\left(P_{01}-P_{02}\right)^{\frac{1}{2}}\left[1+\frac{1}{16}\left(\frac{\Delta p}{\Delta P_{0}}\right)^{2}+\frac{15}{2 \cdot 384}\left(\frac{\Delta p}{\Delta P_{0}}\right)^{4}\right]}
$$

where $\Delta P=P_{01}-P_{02}$ and $Q_{M}$ represent measured flow rate. Since $\Delta p=10^{4} \mathrm{~Pa}$ and $\Delta P_{0}=0,5 \cdot 10^{6} \mathrm{~Pa}$, as the result we obtain

$$
\frac{Q_{M}}{Q_{a}}=\frac{1}{1+\frac{1}{4}\left(\frac{10^{8}}{10^{12}}\right)^{2}}=0,99973
$$

This mean that the actual flow rate is $Q_{a}=0,999973 Q_{M}$

\subsection{Measurement on the reactor}

The pressure drop is measured as a difference between inlet and outlet reactor pressure nozzles. The following relations are valid on the inlet nozzle the pressure is expressed as

$$
P_{1}=P_{01}+\overline{\Delta p} \sin \left(\omega+\delta_{i}\right) t
$$




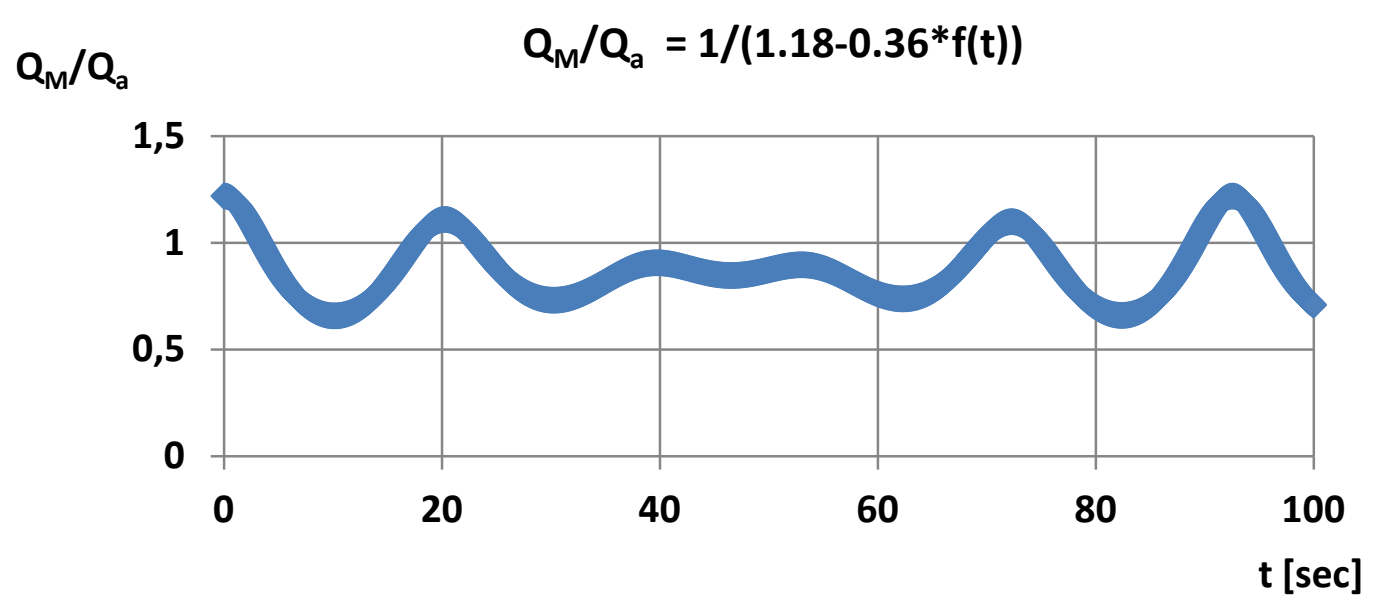

Fig. 2: Ratio $\frac{Q_{M}}{Q_{a}}$ for the reactor

where $\delta_{i}$ is related to the preselected MCP on the outlet nozzle the pressure must be expressed as

$$
P_{2}=P_{02}+\sum_{i=1}^{4} \overline{\Delta p} \sin \left(\omega+\delta_{i}\right) t
$$

since inside the pressure vessel exists pressure pulsations of all fourth MCP.

For the simplification, we will suppose that amplitudes $\overline{\Delta p}$ of all operated MCPs are identical. After simple rearrangements, the following equation takes place

$$
\sum_{i=1}^{4} \sin \left(\omega+\delta_{i}\right) t=4 \sin \omega t \cos \left(\frac{\delta_{1}+\delta_{2}+\delta_{3}+\delta_{4}}{2} t\right) \cos \left(\frac{\delta_{1}+\delta_{2}-\delta_{3}-\delta_{4}}{2} t\right)
$$

and thus

$$
\begin{aligned}
\Delta p & =\left[\left(P_{01}-P_{02}\right)+\overline{\Delta p} \sin \omega_{i} t-4 \overline{\Delta p} \sin \omega_{i} t\left(\cos \frac{\delta_{1}+\delta_{2}+\delta_{3}+\delta_{4}}{2} t * \cos \frac{\delta_{1}+\delta_{2}-\delta_{3}-\delta_{4}}{2} t\right)\right] \\
& =\left[\left(P_{01}-P_{02}\right)+\overline{\Delta p} \sin \omega_{i} t-4 \overline{\Delta p} \sin \omega_{i} t f(\tau)\right]
\end{aligned}
$$

where function $f(\tau)$ represents the beats. In this case equation (4) takes form

$$
Q_{a}=\frac{\bar{K}}{T} \int_{U}^{T} \sqrt{\left(P_{01}-P_{02}\right)+\overline{\Delta p} \sin \omega_{i} t-4 \overline{\Delta p} \sin \omega t f(\tau)} d t
$$

To calculate equation (7) the trinomial expansion must be applied. Performing complicated integration as the result we obtain

$$
\frac{Q_{M}}{Q_{a}}=\frac{1}{1+\frac{4}{\pi} \sqrt{\frac{\overline{\Delta p}}{\Delta P_{0}}}-\frac{8}{\pi} \sqrt{\frac{\overline{\Delta p}}{\Delta P_{0}} f(\tau)}}
$$

The graphical illustration of this function is to be seen in Fig. 2.

\subsection{Measurement on the steam generator}

Measurement of the pressure drop on the SG is specific. Pressure pulsations on the input of the SG are identical with this one on the output. From the mathematical point of view it may be defined as

- $P_{1}=P_{01}+\sum_{i=1}^{4} \overline{\Delta p} \sin \left(\omega+\delta_{i}\right) t$

- $P_{2}=P_{02}+k \sum_{i=1}^{4} \overline{\Delta p} \sin \left(\omega+\delta_{i}\right) t$ 


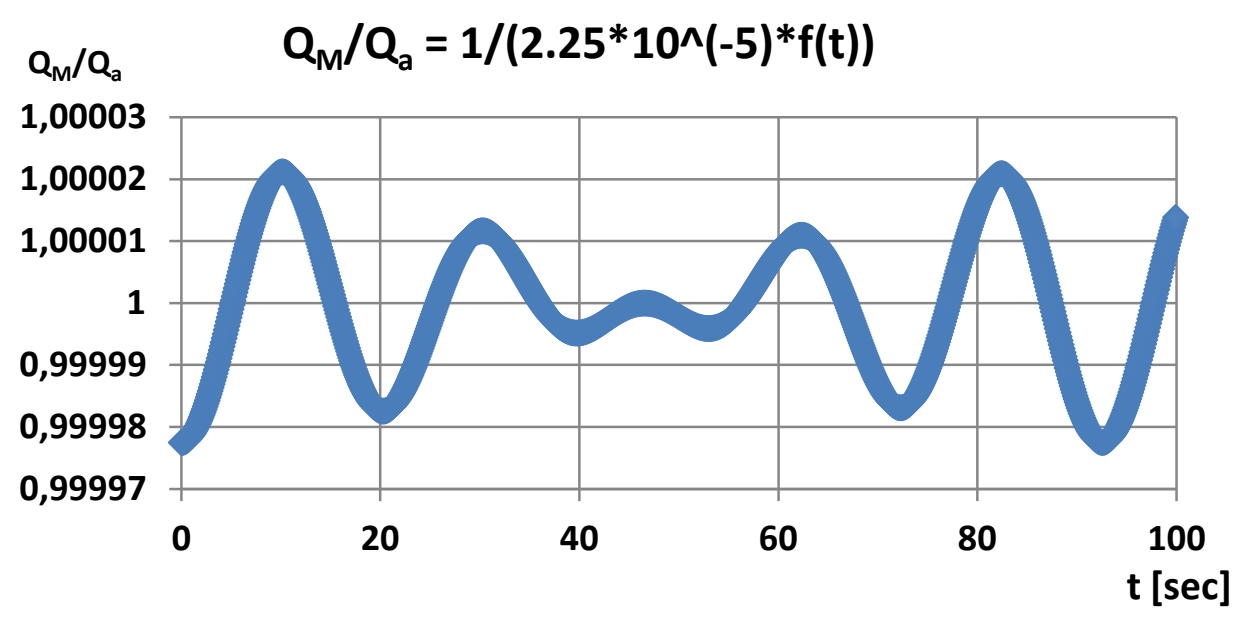

Fig. 3: Ratio $\frac{Q_{M}}{Q_{a}}$ for the steam generator

where $k$ represents damping of the pressure pulsations passing through the SG. As the result, equation (6) changes to

$$
\Delta p=\left[\Delta P_{01}-4 \overline{\Delta p} k \sin \omega t \cos \left(\frac{\delta_{1}+\delta_{2}+\delta_{3}+\delta_{4}}{2} t\right) * \cos \left(\frac{\delta_{1}+\delta_{2}-\delta_{3}-\delta_{4}}{2} t\right)\right]
$$

Putting this equation in equation (4) as the result we obtain after simply rearrangement

$$
\frac{Q_{M}}{Q_{a}}=\frac{1}{1+k \frac{1}{4} \sqrt{\frac{\overline{\Delta p}}{\Delta P_{0}} f(\tau)}}
$$

Graphical illustration for the $k=0.9$ and for the values $\Delta p$ and $\Delta P_{0}$ and $f(\tau)$ is to be seen in Fig 3 .

\section{Conclusions}

In the paper, the influence of the pressure pulsations generated by the MCPs on the measurement of the flow rate of the coolant in primary circuit of the VVER 1000/320 reactor has been derived. Three measurements of the flow rate are obviously performed: on the coolant flow through main circulation pump, through reactor and through the steam generator. Based on the presented theory the following findings may be formulated:

- pressure pulsations nonsignificantly influence measurement of the flow rate on the main circulation pump;

- the effect of pressure pulsations most significantly influence measurement of the flow rate on the reactor. Very interesting is the influence of the beats;

- the effect of the pressure pulsations is negligible if the flow rate is measured on the steam generator. The beats exist as in the case of the reactor but the influence is minimal. It must be kept in the mind that the presented method of the calculation of the errors due to pressure pulsations is only theoretical. Practical applications are under way.

\section{References}

Lindahl E.J. (1946) Pulsation and Its Effect on Flowmeters. Transactions of the ASME, 68, pp. 883-894.

Oppenheim A.K. and Chiton, E.G. (1955) Pulsating flow measurement - A literature survey. Transaction of the ASME, 77, pp. 231-248. 